\title{
Pollution and acne: is there a link?
}

This article was published in the following Dove Press journal:

Clinical, Cosmetic and Investigational Dermatology

19 May 2017

Number of times this article has been viewed

\section{Jean Krutmann' \\ Dominique Moyal ${ }^{2}$ \\ Wei Liu ${ }^{3}$ \\ Sanjiv Kandahari ${ }^{4}$ \\ Geun-Soo Lee ${ }^{5}$ \\ Noppakun Nopadon ${ }^{6}$ \\ Leihong Flora Xiang ${ }^{7}$ \\ Sophie Seité ${ }^{2}$}

'IUF - Leibniz Research Institute for Environmental Medicine, Düsseldorf, Germany; ${ }^{2}$ La Roche Posay Dermatological Laboratories, Asnières, France; ${ }^{3}$ Department of Dermatology, The General Hospital of Air Force, PLA, Beijing, People's Republic of China; ${ }^{4} \mathrm{Dr}$. Kandhari's Skin \& Dental Clinic, New Delhi, India; ${ }^{5}$ Drs. Woo and Hann Skin Center, Yongsan-gu, Seoul, Korea; ${ }^{6}$ Division of Dermatology, Faculty of Medicine, Department of Medicine, Chulalongkorn University, Bangkok, Thailand; ${ }^{7}$ Department of Dermatology, Huashan Hospital, Fudan University, Shanghai, People's Republic of China
Correspondence: Sophie Seité La Roche-Posay Dermatological Laboratories, I 10 Avenue Henri Barbusse, 92600 Asnières, France $\mathrm{Tel}+33$ I4 6886544

Fax +33 I4 6886688

Email sophie.seite@loreal.com
Abstract: In recent years, the critical role that inflammation may play in the development and progression of acne has become increasingly recognized. The prevalence of acne is similar between Asian and Caucasian women, but Asian women have a higher prevalence of inflammatory acne. They also report their symptoms exacerbate during periods of high air pollution. The objective of this study was to review the current evidence that links air pollution to worsening of acne symptoms. Firstly, a group of five Asian and three European scientists with expertise in Dermatology reviewed the current literature and described current acne treatment practices in their countries. During this activity, they identified the need for further epidemiological and clinical research. Secondly, additional studies ensued which provided evidence that acne symptoms might exacerbate in regions of high ambient air pollution. Based on these findings, the authors suggest that people with acne should protect the natural barrier function of their skin with emollients and ultraviolet (UV)A/UVB protection.

Keywords: pollution, acne, Asia, epidemiology, pathophysiology

\section{Introduction}

Acne vulgaris is gradually becoming recognized as an inflammatory disorder of the pilosebaceous unit. ${ }^{1}$ It is a common skin disease with a peak prevalence in teenage years but also occurs in girls pre-menarche and can continue to be a problem in women past adolescence. ${ }^{2}$ Although most epidemiological data in Western countries concern Caucasian skin types, acne is also a significant problem for Asian skin. In one large intercontinental, epidemiological study, the prevalence of clinical acne was reported to be similar between geographical zones, $24 \%$ in Caucasian, $30 \%$ in Asian and $23 \%$ in continental Indian women. However, acne type was found to vary between skin subtypes. Asian women were reported to have a higher prevalence of inflammatory acne than comedonal acne (20\% vs $10 \%)$, compared to Caucasian women, for whom comedonal acne was more prevalent (14\%) than inflammatory acne $(10 \%){ }^{3}$

In recent years, an increasing number of studies indicate a link between skin problems and exposure to airborne pollutants, such as particulate matter (PM), volatile organic compounds, ozone $\left(\mathrm{O}_{3}\right)$, nitrogen dioxide $\left(\mathrm{NO}_{2}\right)$ and sulfur dioxide $\left(\mathrm{SO}_{2}\right) \cdot{ }^{4-7} \mathrm{As}$ a recent example, $\mathrm{NO}_{2}$ exposure has been linked to pigment spot formation on cheeks in both Caucasian and Asian women. ${ }^{8,9}$ Yet, few data are available today concerning the prevalence or exacerbation of acne symptoms and pollution exposure. Some anecdotal evidence emerged during an International Dermatology Conference in Beijing, where 
a survey of dermatologists revealed that $67 \%$ of responders agreed that acne prevalence increases with pollution (personal communication). However, little published evidence was found to support this relationship with the various types of airborne pollutants. One epidemiological study in China conducted in 2007 explored the prevalence of greasy skin in a metropolitan area and found that $25.6 \%$ of participants reported having greasy skin compared with a nonpolluted area. ${ }^{10}$ However, the authors did not evaluate acne signs as such. Two clinical studies conducted in two major cities known to have high pollution levels, Shanghai and Mexico City, found that skin quality changes with chronic exposure to ambient pollution. Skin quality changes were defined as reduced vitamin $\mathrm{E}$ and squalene levels, which are known signs of sebum oxidation. Also, similar to the Chinese study, the Mexican studies found raised sebum levels, but clinical signs of acne were not measured. ${ }^{11}$ Nevertheless, increased sebum is one of the known early, pathophysiological pillars of acne. ${ }^{12}$

In 2014, an Eurasian board of experts published a stateof-the-art review of the current knowledge about the effects of common pollutants on the sensitive skin. ${ }^{13}$ The next step was to further investigate the link between airborne pollution and acne symptoms exacerbation and to be able to propose appropriate measures to protect acne prone skin from further aggravation by pollution. A board of European and Asian scientists with expertise in dermatology gathered in 2015 to discuss the existing scientific evidence. This review summarizes the outcome of that meeting and subsequently initiated studies.

\section{Methods}

The Eurasian board of experts consisted of three European and four Asian scientists with expertise in dermatology, skin biology and environmental medicine. Each expert performed a literature review for research concerning acne prevalence and severity in association with pollution in their country, reviewed the current reports about acne symptom exacerbation and pollution as well as the current knowledge about the pathophysiology of acne and the mechanisms by which airborne pollution interacts with the skin. They were also asked to present their individual clinical experience treating acne in places with high atmospheric pollution.

A further literature review was performed using PubMed. Keyword combinations included air pollution + acne, ozone + acne, skin, $\mathrm{PM}_{10}, \mathrm{PM}_{2.5}$ particulate pollution, ozone, cutaneous disorders + air pollution, environmental stressors, atmospheric pollution, outdoor pollution and sebum. The most recent publications were chosen from key molecular and cellular studies, review articles, epidemiological studies, surveys and exploratory research in human subjects. The objective of the round table meeting was to evaluate the quantity and quality of the literature available. The experts were then to suggest whether further research was required to subsequently prepare a review paper and propose appropriate measures to protect acne prone skin in polluted environments. If further research was required, the board was to identify protocol outlines and workgroups to complete these research needs.

\section{Results \\ Pathophysiology of acne as an inflammatory disease}

The current understanding of the pathophysiology of acne is evolving. Acne is accepted to have a multifactorial etiology, based on three main observations: increased sebum production (under hormonal stimulation), abnormal keratinization of the pilosebaceous duct, and it is becoming increasingly evident that acne is driven by an inflammatory reaction to Propionibacterium acnes. ${ }^{14,15}$

Two early events that occur when lesions appear are hyperseborrhea and follicular obstruction of the pilosebaceous unit. Several comprehensive review papers highlight recent molecular and cellular research that suggest with acne, the quality of sebum excreted on the skin surface changes. This alters the lipid composition and redox ratio on the skin surface. Within the follicular lumen, these qualitative changes in sebum production may result in an overall deficiency in linoleic acid, which disturbs the follicular barrier function. This situation creates a proinflammatory pattern with proinflammatory cytokine production and interleukin (IL)-1 upregulation, ${ }^{16}$ inducing follicular inflammation. ${ }^{15,17,18}$ It has been suggested that IL-1 production may also contribute to hyperkeratinization and the formation of the microcomedone. Also, immune cells (Th1) have been found in perifollicular regions of nonaffected skin in patients with acne, confirming the involvement of the innate immune system. These findings suggest that inflammation may have a critical role in acne development and progression. ${ }^{19}$

One of the lipids produced by human sebaceous glands on the face and torso is squalene. This unsaturated fatty acid represents $\sim 10 \%-15 \%$ of sebum and is readily oxidized by ozone, long ultraviolet (UV) rays and tobacco smoke. ${ }^{20}$ Byproducts produced from squalene peroxidation are known to be comedogenic and also demonstrate proinflammatory properties. ${ }^{18,21}$ High levels of squalene alter the redox balance in the skin leading to an excessive generation of reactive oxygen species (ROS) and a state of oxidative stress. Oxidative stress and nitrosative stress have been shown to 
occur in acne ${ }^{22}$ and may also be the trigger that sets off the acne cascade. ${ }^{23}$

The proliferation of $P$. acnes has long been considered the culprit of the acne cascade, yet this is no longer considered the case, because recent studies have found that $P$. acnes predominates on both normal and disease-associated skins. ${ }^{24}$ However, a recent hypothesis suggests that the blocked pore makes the folliculopilosebaceous unit increasingly hypoxic. As $P$. acnes is known to appreciate anaerobic microenvironments, such as the skin follicle, this situation is perfectly suitable for this usually commensal bacteria to grow. Proliferating $P$. acnes is then recognized by follicular keratinocytes that start releasing inflammatory cytokines in response. The innate immune system is subsequently triggered when the duct ruptures, releasing $P$. acnes into surrounding epidermal tissue, causing pustule formation and potentiating local inflammation..$^{25}$ The innate immune system is thus recruited, helping to drive acne pathogenesis. ${ }^{14}$

Nevertheless, increasing evidence demonstrates that some commensal organisms on human skin regulate the populations of pathogenic strains to maintain homeostasis. For example, Staphylococcus epidermis mediates glycerol fermentation, hence inhibiting $P$. acnes growth. ${ }^{26}$

\section{Pollution as an inflammatory mediator}

Outdoor pollution is a heterogeneous mixture of chemicals and gases emitted into the atmosphere from natural and manmade sources. Those of major concern for the skin include both organic and inorganic compounds found in and on PM and gases such as ozone, nitrogen dioxide $\left(\mathrm{NO}_{2}\right)$ and sulfur dioxide. Unsurprisingly, there are few data on visits for acne during peaks in pollution as many pollution and health studies are performed in accident and emergency departments, where acne patients do not usually present for exacerbation of their symptoms.

There is growing evidence that these pollutants exert their harmful effects by generating oxidative stress by means of ROS and inflammation. However, the exact mechanism is yet to be clarified. Various hypotheses have been proposed to explain how the detrimental effect of pollution starts. Vierkotter et al suggest PMs have an indirect effect by an outside-inside signaling pathway. ${ }^{8} \mathrm{O}_{3}$ has been shown to increase cytochrome p450 activation via the AhR. ${ }^{27}$ Recently, a direct action has been suggested by penetration through the stratum corneum. ${ }^{28}$ Once in the skin, the ROS cause a lipid peroxidation cascade, which stimulates the release of other proinflammatory mediators from keratinocytes and melanocytes, thus establishing a vicious inflammatory cycle, with infiltration of other potentiating cells such as neutrophils and other phagocytic cells. ${ }^{5}$ Polycyclic aromatic hydrocarbons, commonly found in air pollution, are known to also induce an inflammatory response. ${ }^{29-31}$ Finally, AhR activation (MMP, COX2, POMC, MITF) potentiates inflammation, which could theoretically increase acne. ${ }^{32}$

This stress has been suggested to eventually overwhelm the skin's natural defenses depleting its antioxidant capacity, such as vitamins $\mathrm{C}$ and $\mathrm{E}$, contributing to a background inflammatory environment and disrupting the skin barrier function. ${ }^{33,34}$

UV exposure has an additive effect with airborne pollutants and $\mathrm{O}_{3}$. UV irradiation is known to compromise the skin barrier and $\mathrm{O}_{3}$ seems to heighten this phenomenon by disturbing stratum corneum lipid constituents that are known to be critical determinants of the barrier function. UV exposure and $\mathrm{O}_{3}$ have also been found to have an additive effect on antioxidant depletion (vitamin E) and on lipid peroxidation levels, which could, in turn, lead to additional additive effects of these stressors..$^{5,35}$

\section{Acne treatments used in Asia}

Similar to most countries around the world, Asian dermatologists treat acne according to the Global Alliance or American Association of Dermatology guidelines. However, the board members remarked that some medical treatments disrupt skin barrier function. This being particularly problematic in Asian skin, which has been reported to have a higher prevalence and objective skin sensitivity compared with Caucasian skin. ${ }^{36,37}$ Also, there is an increasing awareness of antibiotic resistance due to many years of inappropriate topical antibiotic use and hence the need to find alternative solutions. Board members report a general trend toward using keratolytic (i.e., lipohydroxy acids, glycolic acid, salycilic acid) $)^{38}$ and anti-inflammatory ingredients (i.e., ketones, aldehydes, niacinamide) and sebum regulators, in combination with benzoyl peroxide (BPO) or retinoic acid. In Thailand, it is difficult to implement the Global Alliance guidelines, so they developed their own recommendations. Patients are treated according to severity. They use photodynamic therapy (PDT) lasers to target comedones, but this has been found to be unsatisfactory as acne returns after treatment. In China, the board reports that traditional medicine remains popular for papulopustular acne and is used alongside the Global Alliance Guidelines. PDT (red and blue light) and glycolic acid are also used to treat comedones. In Korea, Chinese traditional medicine is also used, as well as essential oils, tea tree oil and ketone or aldehyde products with an anti-inflammatory activity. 


\section{Additional research}

The first study to be performed following recommendations made by the Expert board was a large, epidemiological study in a dermatology hospital, Beijing, in 2016. Retrospective data were collected over a 2-year period and included 59,530 patients with acne. The authors found that increased ambient air concentrations of some of the most important traffic-and industry-related pollutants were associated with an increased number of outpatient visits for acne vulgaris. These associations were significant for $\mathrm{NO}_{2}$ in a single pollutant model, and $\mathrm{NO}_{2}, \mathrm{PM}_{10}$ and $\mathrm{PM}_{2.5}$ in two pollutant models, which reflect real exposure scenarios more closely (Wei et al, unpublished data, 2017). Also, a second, independent clinical study was performed by La Roche-Posay in Beijing, China, to investigate the relationship between exposure to the most frequent industry- and traffic-related air pollutants, that is, $\mathrm{PM}_{10}$, $\mathrm{PM}_{2.5}, \mathrm{NO}_{2}, \mathrm{SO}_{2}, \mathrm{O}_{3}$ and sebum level as well as the number of acne lesions (inflammatory and noninflammatory lesions). This 8-week study included 64 patients with acne who used the same skin care routine. Sebum rate was measured and acne lesions were counted each week. Air pollution data were obtained from the Beijing municipal environmental monitoring center. Increased ambient concentrations of $\mathrm{PM}_{2.5}$, $\mathrm{PM}_{10}$ and $\mathrm{NO}_{2}$ were associated with increased sebum secretion and a higher number of acne lesions. Higher ambient $\mathrm{O}_{3}$ concentrations were significantly associated with lower sebum rate and lower numbers of lesions (inflammatory and noninflammatory lesions) (unpublished data). Interestingly, both studies found that higher ambient $\mathrm{SO}_{2}$ concentrations were associated with fewer outpatient visits, lower sebum rates and lower numbers of both inflammatory and noninflammatory lesions. Together, these two studies indicate that exposure to air pollutants may aggravate acne vulgaris and emphasize that not all air pollutants have the same effect on acne skin.

Finally, L'Oréal Research and Innovation performed another study in two industrial areas in Asia to investigate the effect of ambient pollution on skin, including acne characteristics and severity. This study was retrospective in nature. It was observed that acne prevalence was significantly different between the two populations.

These results further support the pathophysiological links between acne prevalence and high ambient pollution levels.

\section{Further research}

Based on the literature searches and these three studies the authors suggest that more research is needed to better understand the link between acne and air pollution. In particular, there is a clear need for mechanistic studies in order to better understand the existing epidemiological evidence for this link. In particular, the board suggested to further investigate the relationship between pollution exposure and squalene oxidation. Also, more research would be useful to understand the change in composition and quality of sebum with pollution.

\section{Discussion and suggestions for appropriate protective measures}

There is little data available to make strong recommendations for adjunctive skin care in acne management in polluted environments. Rocha et al recommends emollients and sunscreen in addition to drug treatment and suggests that patients be advised not to manipulate lesions. ${ }^{1}$ The natural skin barrier can be altered by using detergents that disrupt the skin lipid barrier, and induce the loss of antimicrobial peptides. This has been shown to allow $S$. epidermis to proliferate and attenuate the innate immune response. ${ }^{39}$ In light of the data available, the board supports the goal of dermocosmetic protection to reinforce skin barrier function. This prevents further provoking the inflammatory cycle and in turn associated problems such as aggravated disease severity and postinflammatory pigmentation.

1. A skin care product dedicated to acne patients may provide a protective barrier from pollution, restore microbiome equilibrium to prevent overabundant bacteria (including $P$. acnes) colonization and control disease severity and postinflammatory pigmentation, which is a concern in this population.

2. Some acne medication such as isotretinoin, topical retinoids and BPO dry the skin. Patients using these products should apply appropriate moisturizers as complimentary therapy to control skin dryness and restore skin barrier function.

3. Appropriate cleansers that do not further disturb the natural barrier function are suggested to eliminate pollution on the skin surface. Avoid using facial or body scrubs. Cleanse the skin well. Use mild medical cleansers.

4. Certain acne medications are known to cause druginduced phototoxicity. Sun protection should be encouraged: ensure the formulation has sufficient UVA and UVB protection, and is noncomedogenic and has a texture adapted to oily skin that provides moisture, while reducing sebum and facial shine.

\section{Acknowledgments}

The authors acknowledge Amy Whereat, Speak the Speech Consulting, France, for providing medical writing support for this manuscript. 
La Roche Posay Dermatological Laboratories, Asnières, France, provided funding for the board meeting and medical writing support to draft and edit the manuscript.

\section{Disclosure}

S Seité and D Moyal are employees of La Roche Posay Dermatological Laboratories, Asnières, France. The authors report no other conflicts of interest in this work.

\section{References}

1. Rocha MA, Costa C, Bagatin E. Acne vulgaris an inflammatory disease even before the onset of clinical lesions. Inflam Allergy Drug Targets. 2014;13(3):162-167.

2. Collier CN, Harper JC, Cafardi JA, et al. The prevalence of acne in adults 20 years and older. J Am Acad Dermatol. 2008;58(1):56-59.

3. Perkins AC, Cheng CE, Hillebrand GG, Miyamoto K, Kimball AB. Comparison of the epidemiology of acne vulgaris among Caucasian, Asian, Continental Indian and African American women. J Eur Acad Dermatol Venereol. 2011;25(9):1054-1060.

4. Larrieu S, Lefranc A, Gault G, et al. Are the short-term effects of air pollution restricted to cardiorespiratory diseases? Am J Epidemiol. 2009; 169(10):1201-1208.

5. Valacchi G, Sticozzi C, Pecorelli A, Cervellati F, Cervellati C, Maioli E. Cutaneous responses to environmental stressors. Ann NY Acad Sci. 2012; 1271:75-81.

6. Xu F, Yan S, Wu M, et al. Ambient ozone pollution as a risk factor for skin disorders. Br J Dermatol. 2011;165(1):224-225.

7. Kim J, Kim EH, Oh I, et al. Symptoms of atopic dermatitis are influenced by outdoor air pollution. J Allergy Clin Immunol. 2013;132(2):495-498 e491.

8. Vierkotter A, Schikowski T, Ranft U, et al. Airborne particle exposure and extrinsic skin aging. J Invest Dermatol. 2010;130(12):2719-2726.

9. Huls A, Vierkotter A, Gao W, et al. Traffic-related air pollution contributes to development of facial lentigines: further epidemiological evidence from Caucasians and Asians. J Invest Dermatol. 2016;136(5): 1053-1056.

10. Nouveau-Richard S, Zhu W, Li YH, et al. Oily skin: specific features in Chinese women. Skin Res Technol. 2007;13(1):43-48.

11. Lefebvre MA, Pham DM, Boussouira B, Bernard D, Camus C, Nguyen QL. Evaluation of the impact of urban pollution on the quality of skin: a multicentre study in Mexico. Int J Cosmet Sci. 2015;37(3):329-338.

12. Mourelatos K, Eady EA, Cunliffe WJ, Clark SM, Cove JH. Temporal changes in sebum excretion and propionibacterial colonization in preadolescent children with and without acne. Br J Dermatol. 2007;156(1):22-31.

13. Krutmann J, Liu W, Li L, et al. Pollution and skin: from epidemiological and mechanistic studies to clinical implications. J Dermatol Sci. 2014; 76(3):163-168.

14. Dreno B, Gollnick HP, Kang S, et al. Understanding innate immunity and inflammation in acne: implications for management. J Eur Acad Dermatol Venereol. 2015;29(Suppl 4):3-11.

15. Makrantonaki E, Ganceviciene R, Zouboulis C. An update on the role of the sebaceous gland in the pathogenesis of acne. Dermatoendocrinol. 2011;3(1):41-49.

16. Elias PM, Brown BE, Ziboh VA. The permeability barrier in essential fatty acid deficiency: evidence for a direct role for linoleic acid in barrier function. J Invest Dermatol. 1980;74(4):230-233.

17. Ottaviani M, Alestas T, Flori E, Mastrofrancesco A, Zouboulis CC, Picardo M. Peroxidated squalene induces the production of inflammatory mediators in $\mathrm{HaCaT}$ keratinocytes: a possible role in acne vulgaris. J Invest Dermatol. 2006;126(11):2430-2437.
18. Zouboulis CC, Jourdan E, Picardo M. Acne is an inflammatory disease and alterations of sebum composition initiate acne lesions. J Eur Acad Dermatol Venereol. 2014;28(5):527-532.

19. Harvey A, Huynh TT. Inflammation and acne putting the pieces together. J Drugs Dermatol. 2014;13(4):459-463.

20. Pham DM, Boussouira B, Moyal D, Nguyen QL. Oxidization of squalene, a human skin lipid: a new and reliable marker of environmental pollution studies. Int J Cosmet Sci. 2015;37(4):357-365.

21. Tanghetti EA. The role of inflammation in the pathology of acne. J Clin Aesthet Dermatol. 2013;6(9):27-35.

22. Al-Shobaili HA, Alzolibani AA, Robaee AA, Meki AR, Rasheed Z. Biochemical markers of oxidative and nitrosative stress in acne vulgaris: activity. J Clin Lab Anal. 2013; 27(1):45-52.

23. Bowe WP, Patel N, Logan AC. Acne vulgaris the role of oxidative stress and the potential therapeutic value of local and systemic antioxidants. J Drugs Dermatol. 2012;11(6):742-746.

24. Fitz-Gibbon S, Tomida S, Chiu BH, et al. Propionibacterium acnes strain populations in the human skin microbiome associated with acne. J Invest Dermatol. 2013;133(9):2152-2160.

25. Danby FW. Ductal hypoxia in acne: is it the missing link between comedogenesis and inflammation? J Am Acad Dermatol. 2014;70(5):948-949.

26. Wang Y, Kuo S, Shu M, et al. Staphylococcus epidermidis in the human skin microbiome mediates fermentation to inhibit the growth of Propionibacterium acnes: implications of probiotics in acne vulgaris. Appl Microbiol Biotechnol. 2014;98(1):411-424.

27. Afaq F, Zaid MA, Pelle E, et al. Aryl hydrocarbon receptor is an ozone sensor in human skin. J Invest Dermatol. 2009;129(10):2396-2403.

28. Magnani ND, Muresan XM, Belmonte G, et al. Skin damage mechanisms related to airborne particulate matter exposure. Toxicol Sci. 2016; 149(1):227-236.

29. Nel AE, Diaz-Sanchez D, Ng D, Hiura T, Saxon A. Enhancement of allergic inflammation by the interaction between diesel exhaust particles and the immune system. J Allergy Clin Immunol. 1998;102(4 Pt 1): 539-554.

30. Casillas AM, Hiura T, Li N, Nel AE. Enhancement of allergic inflammation by diesel exhaust particles: permissive role of reactive oxygen species. Ann Allergy Asthma Immunol. 1999;83(6 Pt 2): 624-629.

31. Yun YP, Joo JD, Lee JY, et al. Induction of nuclear factor-kappaB activation through TAK1 and NIK by diesel exhaust particles in L2 cell lines. Toxicol Lett. 2005;155(2):337-342.

32. Ahn EK, Yoon HK, Jee BK, et al. COX-2 expression and inflammatory effects by diesel exhaust particles in vitro and in vivo. Toxicol Lett. 2008;176(3):178-187.

33. Mancebo SE, Wang SQ. Recognizing the impact of ambient air pollution on skin health. J Eur Acad Dermatol Venereol. 2015;29(12):2326-2332.

34. Lee CW, Lin ZC, Hu SC, et al. Urban particulate matter down-regulates filaggrin via COX2 expression/PGE2 production leading to skin barrier dysfunction. Sci Rep. 2016;6:27995.

35. Pillai S, Oresajo C, Hayward J. Ultraviolet radiation and skin aging: roles of reactive oxygen species, inflammation and protease activation, and strategies for prevention of inflammation-induced matrix degradation - a review. Int J Cosmet Sci. 2005;27(1):17-34.

36. Berardesca E, Farage M, Maibach H. Sensitive skin: an overview. Int J Cosmet Sci. 2013;35(1):2-8.

37. Lee E, Kim S, Lee J, Cho SA, Shin K. Ethnic differences in objective and subjective skin irritation response: an international study. Skin Res Technol. 2014;20(3):265-269.

38. Saint-Léger D, Lévêque JL, Verschoore M. The use of hydroxy acids on the skin: characteristics of C8-lipohydroxy acid. J Cosmet Dermatol. 2007;6(1):59-65.

39. Muszer M, Noszczynska M, Kasperkiewicz K, Skurnik M. Human Microbiome: when a friend becomes an enemy. Arch Immunol Ther $\operatorname{Exp}$ (Warsz). 2015;63(4):287-298. 


\section{Publish your work in this journal}

Clinical, Cosmetic and Investigational Dermatology is an international, peer-reviewed, open access, online journal that focuses on the latest clinical and experimental research in all aspects of skin disease and cosmetic interventions. This journal is included on PubMed. The manuscript management system is completely online and includes a very quick and fair peer-review system, which is all easy to use. Visit http://www.dovepress.com/testimonials.php to read real quotes from published authors 